\title{
EL DIARIO PERSONAL DEL ALUMNO COMO INSTRU- MENTO DE REFLEXIÓN EN EXPRESIÓN CORPORAL
}

\section{Autores}

TERESA GARCÍA SANEMETERIO

PERLA MORENO ARROYO

FACULTAD DE CIENCIAS DEL DEPORTE DELA UNIVERSIDAD

DE EXTREMADURA

\section{RESUMEN}

La reflexión del alumno como condicionante de la construcción de su conocimiento y como factor fundamental en la formación inicial de los futuros docentes, es el punto de partida del presente artículo, resultado final de un trabajo iniciado el año 2000 en la Facultad de Ciencias del Deporte de la Universidad de Extremadura con los alumnos de la asignatura de Expresión Corporal.

La finalidad principal del presente estudio fue de tipo formativo: se pretendió desarrollar la capacidad reflexiva de los alumnos. Para ello se les solicitó la elaboración de un diario personal sobre la asignatura, un documento íntimo en el que reflejaran sus reflexiones a lo largo del proceso de enseñanza-aprendizaje de dicha materia. La importancia de dicho documento se manifestó finalmente, además, para la profesora, como un útil instrumento de recogida de datos procedentes de los alumnos.

Tras el análisis de los datos, se presentan las conclusiones pertinentes y las principales sugerencias que podrían orientar investigaciones futuras para el desarrollo de la reflexión en el alumnado.

La reflexión del alumno en la construcción del conocimiento y en los programas de formación inicial de los docentes

Los principios fundamentales en los que se sustenta el sistema educativo, constructivismo y aprendizaje significativo, justifican la necesidad de implicación, predisposición, y participación creativa y crítica (aspectos, éstos dos últimos incluidos en la concepción del proceso activo de educación, según García Aretio, 1989)، por parte del aprendiz.

De acuerdo con Pozo (2001), el aprendizaje constructivo se relaciona con un aprendizaje autónomo, centrado en el interés del alumno por comprender, lo cual supone una gran implicación personal por parte del aprendiz. Según Pozo (2001:165), los distintos niveles de construcción del conocimiento requieren que "... el aprendiz 'tome conciencia' o reflexione activamente sobre los conflictos entre sus conocimientos previos y la nueva información". A pesar de que en ciertas situaciones se actúa de acuerdo con automatismos adquiridos, la reflexión sobre rutinas de actuación o sobre los productos y procesos de aprendizaje, en palabras de Pozo (2001:204), "... deberá sin duda mejorar ese aprendizaje".
La concepción constructivista entiende la enseñanza y el aprendizaje como procesos interactivos inseparables $e$ incomprensibles de forma aislada, (Marchesi y Martín, 1999). La enseñanza, en este enfoque constructivo, debe ayudar de forma constante a los alumnos en su proceso de aprendizaje. Según Marchesi y Martín (1999:388,389), "La función del docente, desde este punto de vista, es facilitar la actividad mental de los alumnos que les permita construir nuevos conocimientos a partir de la reconstrucción y reorganización de los que ya poseen". Entre algunas de las propuestas de intervención pedagógica, acordes con la concepción indicada, sugeridas por Coll (1987) podemos destacar: generar conflictos y resolución de conflictos; plantear/confrontar diferentes puntos de vista; plantear un trabajo cooperativo; considerar los errores o el resultado de una actividad.

Aparte de esta importancia concedida a la intervención y reflexión por parte del alumno durante el proceso de enseñanza-aprendizaje, y a la necesidad de llevar a cabo una actuación docente que facilite o contribuya a la reconstrucción y reorganización de los conocimientos, cuando los alumnos/aprendices son profesores en formación, los programas formativos tratan de incidir también en la reflexión.

La formación del profesorado la entendemos como un proceso continuo (Carreiro da Costa, 1996), en el que pueden intervenir numerosos agentes (Debesse, 1982), y 
cuyo fin es mejorar la calidad de la enseñanza que reciben los alumnos, mediante la formación de los profesores en el conocimiento de determinados contenidos, el dominio de las destrezas docentes y el desarrollo de una actitud crítica y reflexiva. Autores como Pérez (1992, 1995), Moral (1998), Ramos (1999), consideran fundamental formar docentes críticos y reflexivos, capaces de ir contribuyendo con su reflexión "sobre la acción" y "en la acción", (Schön, 1983), a la mejora de su calidad docente.

Camerino y Buscá (2001:235) incluyen en el modelo reflexivo, empleado en la formación inicial, los siguientes procesos:

Autocrítica sobre las percepciones, pensamientos y acciones realizadas en el contexto profesional.

Análisis de las decisiones tomadas, experiencias o productos obtenidos.

Proceso de pensamiento que posibilite intervenciones adecuadas a las diferentes situaciones.

En consonancia con las ideas que venimos exponiendo, partiendo de los principales objetivos de la formación del profesorado de educación física, y centrándonos en la formación crítica y reflexiva de los docentes, proponemos siguiendo a Carreiro da Costa (1996) una serie de sugerencias y estrategias a emplear en los programas de formación del profesorado de educación física.

Incentivar a los futuros profesores a que se cuestionen sus concepciones acerca de la enseñanza y analicen y reinterpreten sus experiencias anteriores. Es decir, que hagan una valoración crítica sobre sus experiencias en educación física escolar; que analicen de forma crítica la realidad actual de las prácticas de educación física escolar y las condiciones de ejercicio profesional; que analicen críticamente las orientaciones y objetivos del curso, relacionándolos con las exigencias profesionales futuras. Para ello, en los programas de formación se pueden emplear estrategias como: simulación, indagación, estudio de casos, historias de vida, análisis de la enseñanza, supervisión clínica, etc.

Formar profesores reflexivos, que se cuestionen su actuación, que tengan curiosidad, interés por mejorar, que sean capaces de distinguir entre una adecuada y una inadecuada práctica educativa. En los programas de formación se pueden emplear las siguientes estrategias: investigación-acción, análisis de enseñanza, autoanálisis, historias de vida, estudio de casos, técnica de incidentes críticos, formación recíproca, etc.

El estudio que ahora presentamos se ubica en la asignatura de Expresión Corporal, incluida en el Plan de Estudios para la obtención de la Licenciatura en Ciencias de la Actividad Física y del Deporte por la Universidad de Extremadura. La intención del mismo es mostrar las diferentes estrategias formativas empleadas para fomentar la reflexión de los aprendices, profesionales en formación inicial. Con dicha finalidad, tras exponer las dos principales estrategias planteadas durante el desarrollo de la asignatura, nos centramos en el análisis del diario del alumno, abordando aspectos relativos a la profundidad de la reflexión y al contenido o temática tratados en el mismo.
Estrategias de reflexión

Como hemos visto hasta ahora, uno de los objetivos principales de nuestra intervención investigadora y docente es fomentar la capacidad reflexiva de los alumnos, y que ésta pueda redundar positivamente tanto en el proceso de aprendizaje de los contenidos propios de la Expresión Corporal, como en su formación de futuros docentes. Para ello hemos aplicado dos estrategias principales, que pasamos a explicar a continuación.

a) Reflexión durante la práctica: esta estrategia de reflexión consistió en hacer, en determinados momentos de la sesión, un inciso para la reflexión en común, con el fin de plantear cuestiones que considero interesantes. En la reflexión se trata de que sean los mismos alumnos los que lleguen a las conclusiones pertinentes. El papel de la profesora consiste en hacer hincapié en las cuestiones relevantes, guiando la reflexión de los alumnos en la dirección oportuna. Destacamos aquí dos tipos de cuestiones:

Cuestiones de forma: englobamos en este apartado las principales ideas de las preguntas lanzadas por la profesora en los momentos de la práctica, que podemos sintetizar en los siguientes puntos:

la utilización del espacio en todas sus dimensiones y la relación corporal con él

la adopción de determinadas formas y/o diseños corporales

la relación con el fondo musical empleado (si es que lo ha habido)

las cuestiones relacionadas con los distintos agrupamientos de los alumnos.

Cuestiones de fondo o «personales»: este apartado lo constituyen las ideas que expresan las vivencias, sentimientos, o sensaciones experimentadas por los alumnos durante las sesiones. Para entender estas cuestiones necesitamos aceptar un punto de partida, y es que la implicación personal, subjetiva del alumno, en Expresión Corporal, a diferencia de otras asignaturas, es inherente al propio desarrollo de la materia. No podemos, no obstante, confundir esta premisa con la invasión de la intimidad de los participantes, que deben ser siempre los directores de la práctica, y quienes tomen la decisión, en última instancia, de determinar hasta dónde llegar en la exposición de su intimidad.

b) Reflexión después de la práctica: el segundo tipo de estrategia empleada para potenciar la reflexión es el objeto de estudio del presente trabajo, los diarios personales de los alumnos. Los alumnos debían elaborar, después de cada sesión, un documento en el que reflejaran sus pensamientos. Este apartado queda reflejado en el siguiente punto: las características y principales ventajas de los diarios personales de los alumnos, y los datos obtenidos en el análisis del caso que nos ocupa. 


\section{Los diarios personales}

\section{El diario del alumno como estrategia reflexiva}

Los diarios son documentos personales en los que el redactor del mismo, recoge sus pensamientos, creencias estados de ánimo, sensaciones, reflexiones, (Colás, 1992).

El empleo que nosotros hacemos del diario en la investigación que ahora presentamos, cumple dos funciones principales:

Actuar como estrategia formativa. Rodríguez (1994, 1997), considera que la propia elaboración de diarios fomenta por sí misma la capacidad de reflexión del sujeto que lo realiza. De hecho, el simple hecho de escribir ya es útil para el alumno, tal y como reconocen Zabalza y cols., citados por Sicilia (1999:29):

«El mero hecho de escribir ayuda a estimular la conciencia y el autoanálisis a través de la introspección... Supone estructurar, realizar procesos complementarios de síntesis-análisis... El mero hecho de escribir es ya positivo».

Servir como instrumento de recogida de datos, enfocado hacia los tres ámbitos indicados por Hopkins (1989:84): aportan la perspectiva del alumno sobre la enseñanza que recibe (Sicilia, 1999); informan sobre el clima general de la clase; posibilitan la triangulación (Sicilia, 1999).

Esta doble función del diario, ha sido empleada en diversas investigaciones desarrolladas en la formación de profesores de Educación Física, así: Del Villar (1993); Fraile (1993); Romero (1995); Medina (1995); Ramos (1999).

A las diferentes posibilidades de empleo del diario del alumno en investigación educativa, indicados anteriormente, Sicilia $(1999: 28,29)$ añade, su utilidad para:

"Democratizar la investigación", al tener en cuenta la opinión de personas a las que normalmente no se les suele preguntar.

$\square$ Aportar información al profesor sobre aspectos relacionados con el aprendizaje de los alumnos.

De forma concreta, en nuestro estudio, mediante el empleo del diario del alumno como estrategia formativa pretendemos que el alumno tome conciencia de su propia actuación durante las sesiones de expresión corporal y que mejore progresivamente su capacidad de reflexión.

En otro sentido, con el uso del diario como instrumento de investigación queremos acceder al contenido de la reflexión del alumno, así como a la profundidad con la que los alumnos reflexionan.

De acuerdo con el uso previsto de este instrumento nos hemos decantado por el empleo de un diario abierto, el cual, según Latorre y González (1987) presenta un amplio grado de libertad en cuanto a su redacción, no precisa preparación específica y relata el clima general del aula. Como orientación para la elaboración del diario a los alumnos simplemente les aportamos unas notas introductorias generales. El contenido de dicha introducción lo presentamos a continuación:

El presente diario pretende recoger tu pensamiento. En él debes de tratar de reflexionar y analizar con sinceridad tus vivencias como alumno de la asignatura durante las diferentes sesiones, haciendo referencia a todo aquello que te parezca reseñable, tanto en lo que respecta a tí mismo como a tus compañeros, a la profesora, o a las propuestas prácticas que se realicen: ideas, problemas, inquietudes, preocupaciones, creencias, expectativas, críticas, sugerencias, etc.

Debes elaborar el diario después de cada sesión, procurando que pase el menor tiempo posible entre el final de la clase y el momento de escribir.

Se consideran válidas todas las opiniones, siempre que sean justificadas.

Nótese la referencia al momento de redacción del diario, lo más próximo posible a la sesión, para evitar el olvido y reflejar de la forma más fiel posible las sensaciones vividas durante la misma.

El uso de este tipo de diarios abiertos, sin embargo, no está exento de inconvenientes, como los indicados por Latorre y González (1987:23): suele ser subjetivo; depende del sujeto que lo realiza. En nuestra investigación, su empleo posibilita el acceso al pensamiento del alumno, sin limitar o condicionar su contenido, aspecto necesario para poder analizar el contenido de la reflexión.

Seránecesario, además, tener en cuenta también el momento en que se realizan otras actividades formativas, con la intención de que no interfieran o condicionen el contenido del diario o los aspectos sobre los que se reflexionan.

\section{Análisis de los datos obtenidos en los diarios}

\section{a) Presentación de la muestra y del grupo de trabajo}

Los documentos analizados son los diarios personales de los alumnos del primer curso de la Facultad de Ciencias del Deporte de Cáceres durante los cursos 1999/ 2000 y 2000/2001. La elaboración de los diarios que analizamos forma parte de los requisitos para la evaluación de la asignatura de Expresión Corporal, materia troncal cuatrimestral de 4.5 créditos.

El nivel de experiencia previa de los alumnos en esta materia es prácticamente nulo, tanto en la práctica de actividades relacionadas con la Expresión Corporal como en la realización de documentos personales o diarios.

\section{b) Consideraciones personales}

Las referencias positivas sobre las actividades, sobre la metodología empleada, y los comentarios sobre los objetivos que los alumnos fueron consiguiendo a lo largo del curso - aumento del grado de confianza con el grupo, y mejoría de la capacidad de utilizar el cuerpo y el movimiento de forma expresiva, principalmente-constituyen el grueso de las opiniones personales extraídas de los documentos analizados.

En el siguiente cuadro exponemos algunos de los testimonios más llamativos y/o representativos, agrupados en seis categorías diferentes, directamente relacionadas con algunos de los objetivos principales de la asignatura. 
REFERENCIAS A...

\section{EJEMPLOS DE TESTIMONIOS}

\section{REFERENCIAS A...}

\section{TRABAJO GRUPAL}

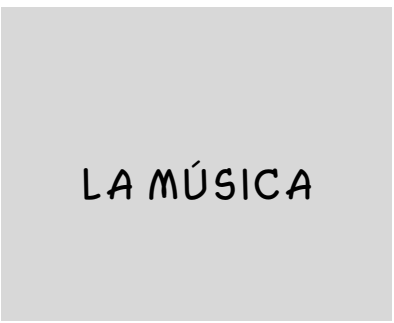

CONTACTO CORPORAL

\section{DESINHIBICIÓN}

AUTOESTIMA

RELAJACIÓN

\section{EJEMPLOS DE TESTIMONIOS}

"Resulta curioso ver como hay uno al que más se le oye, otro al que se le ocurre la mejor idea, otro que lo organiza la idea del anterior, otro que no dice nada, etc., lo que resulta una réplica de la sociedad"

"Creo que todos deberíamos tener mas paciencia con los demás pero algunas cosas empiezan a estar claras, algunos del grupo se empeñan en mandar y eso no sienta demasiado bien"

"Nosotros cuatro damos ideas que no tienen porqué ser las definitivas, sino que pueden y deben ser discutidas, y los demás se callan y dicen que sí a todo. Estas cosas no las entiendo"

"En el grupo me puse imperativo, diciendo cómo lo teníamos que hacer y marcando los tiempos en voz alta, y al final no sirvió de nada, he quedado como el "malo" que llama la atención"

"Como en los demás ejercicios por grupos, hubo unos que colaboramos y otros que esperaban a que les dijésemos lo que tenían que hacer"

"Aunque la comparación sea un tanto extraña (referente a una danza), es como el grito que yo por ejemplo hago con mi equipo en los vestuarios antes de salir a jugar, ambas cosas tiene en definitiva la misma finalidad, unir al grupo"

"He notado la mayor facilidad que tengo para expresar ideas cuando estoy en un grupo de varias personas"

Sensaciones de estímulo, de activación, de sensibilización, o incluso de protección: los testimonios recopilados en los diarios sobre los efectos producidos por la música son siempre positivos:

"La música me estimula, y además me hace sentirme más protegido cuando me pierdo..."

"La música la considero una pieza clave porque transmite sensaciones que sin ella no podríamos sentir"

"Me sigue llamando la atención que nuestros movimientos sigan a la música, ¿o es la música la que sigue a nuestros movimientos?"

"Me pareció que al principio, cuando no se puso ninguna consigna sobre el contacto corporal adoptábamos posiciones mas variadas, pero una vez que se dijo que no hubiera contacto éstas se hicieron quizá más repetitivas o menos variadas, era como si no supiéramos colocarnos de distintas maneras sin tocarnos"

"Había que adivinar en qué posición estaba tu compañero, con los ojos cerrados, solo por el tacto. Cuando la profesora lo explicó, yo temblé, porque mi pareja era una chica"

"Estoy preparándome para lo peor, puesto que el tema de la desinhibición no lo tengo muy controlado, pero quiero ponerme un poco a prueba y verme en situaciones difíciles para ver cómo reacciono"

"Me ha tocado dirigir un grupo de chicos y chicas... No me ha gustado nada y no era vergüenza sino más bien miedo a lo que pudieran pensar de mí, por si no les resultaba divertida..."

"Yo pensaba que este tema lo tenía más controlado, porque no soy una persona tímida, pero ya he visto que no es así, y me he sentido muy expuesta" (tras un ejercicio de realización individual con presencia de compañeros como observadores externos)

"Comprobar que los demás se lo toman en serio me hace sentirme más segura, y entonces me da menos vergüenza"

"La creación de estos 16 pasos ha provocado en mí una sensación de confianza total y una elevación de la autoestima, ya que es totalmente distinto a reproducir algo que ya está elaborado"

"Hoy el ejercicio era voluntario. Después de mucho pensármelo he conseguido dar el paso y he salido a hacerlo. ¡Qué bien me he sentido después!"

"Mis sensaciones con respecto a las danzas de relajación son siempre las mismas, mucha relajación y una sensación de tranquilidad inmensa"

"Ya no oía a la profesora, ni sabía en qué posición me había quedado. Al acabar, me ha parecido que me despertaba de un largo sueño" 


\section{Conclusiones}

En el siguiente apartado pasamos a explicar las dos principales conclusiones obtenidas tras el análisis de los datos recopilados. Estas conclusiones se refieren a dos aspectos fundamentales: la consideración de los diarios como instrumento de reflexión del alumno y la influencia de la evaluación en la elaboración de dichos documentos.

\section{El nivel de reflexión}

La primera impresión que surge tras el análisis de los diarios personales de los alumnos es de una cierta desilusión, ya que el nivel de reflexión en los documentos, no es elevado en la mayoría de los casos: los diarios se centran a menudo en describir los ejercicios, no llegando, la mayoría de las veces, a profundizar en las vivencias que éstos les han supuesto. Los comentarios personales se refieren casi siempre a puntos ya tratados durante la sesión, sobre los que la profesora intentaba incidir y planteados directamente por ella, aspectos que los alumnos reproducen después casi literalmente en el diario.

A este respecto es curioso constatar, sin embargo cómo muchos de los casos de los diarios de mayor nivel de reflexión pertenecen a alumnos que no conocían la asignatura ni tenían ningún tipo de vivencia previa cercana a la expresión corporal. Merece la pena destacar los casos de algunos alumnos que en un principio temían la asignatura por no considerarse en absoluto aptos para ella, o incluso la consideraban despectivamente, y que sin embargo sufrieron una gran evolución, de jando constancia en sus diarios personales de este cambio, y de la aceptación de una nueva posibilidad corporal que ya no les resultaba tan ajena.

Por el contrario, los alumnos más hábiles en la práctica, aceptados como tal por la totalidad del grupo -así quedaba de manifiesto al ser los alumnos más requeridos en el momento de formar grupos, por ejemplo-, no elaboran sin embargo documentos con el grado de reflexión deseado. La evolución y el resultado de los diarios personales son, en ellos, mucho menor. Esto nos hace cuestionarnos lo siguiente: los alumnos más hábiles en la práctica, ¿quizás se conforman con destacar en la "ejecución"? ¿La reflexión que conlleva la elaboración de un documento de este tipo es quizás considerada como algo secundario, o accesorio, siempre subordinada al nivel de la habilidad motriz?

Merece la pena destacar, en cualquier caso, algunos testimonios favorables a la realización de los diarios, que reconocen la eficacia de este instrumento como mediador de la reflexión y el aprendizaje:

"He de decir que me parece muy interesante y positivo que se me dé la oportunidad de expresarme en un trabajo que además te hace reflexionar constantemente sobre la asignatura".

"Solo me queda decir que ha sido un placer hacer el diario. Me parece una buena didáctica, me trae buenas sensaciones...".

\section{Influencia de la evaluación}

Es bastante común encontrar testimonios que sugieren el esfuerzo de los alumnos por superar la tarea de elaboración del diario, ya que constituía un apartado puntuable en la evaluación de la asignatura de Expresión Corporal. Constantemente dichos testimonios demuestran hasta qué punto los alumnos se vieron afectados por este condicionante $y$, en nuestra opinión, en algunas ocasiones incluso limitando su expresión más sincera.

Muchas veces encontramos, además, un evidente "refugio" de los alumnos en datos más académicos, más fríos, en lugar de centrarse en reflexiones personales ya que, pensamos, no es habitual la identificación de lo personal, lo íntimo, o subjetivo, con la evaluación, a pesar de que las directrices que se dieron para la elaboración del documento fueron claras en este sentido.

No obstante aparecieron también numerosas referencias, casi siempre en las reflexiones de las últimas sesiones del curso, en las que los alumnos reconocían su evolución en este aspecto: "A principio de curso escribía el diario sin ganas, porque había que hacerlo para aprobar, y sin embargo ahora me parece que la clase no me serviría para nada si no escribiera sobre ella".

\section{Propuesta de alternativas y sugerencias}

El análisis de los datos recopilados en el presente trabajo nos orienta en la elaboración de líneas de trabajo en las que seguir profundizando en futuras ocasiones.

La inexperiencia previa de los alumnos en la elaboración de documentos de reflexión personal es el condicionante que nos hace pensar en tres sugerencias principales:

1. Por un lado, nos parece oportuno sugerir una extensión mínima (al menos una cara) de escritura reflexiva tras cada una de las sesiones, ya que nos parece positivo el simple hecho de escribir, pues obligamos de este modo al alumno a un esfuerzo de comprensión y análisis tras la práctica.

2. Por otro lado, se hace patente la necesidad de realizar una entrega intermedia del documento a mitad de cuatrimestre, para su corrección y reorientación, si es necesario, buscando siempre la profundidad de la reflexión personal sobre las experiencias vividas.

3. Y finalmente, pensamos, sería una buena alternativa orientar los diarios de alguna forma, mediante preguntas directas e indicaciones específicas de los temas a tratarya que la elaboración totalmente libre, en nuestro caso, ha supuesto una dificultad añadida para los alumnos. Así, podríamos destacar, antes y durante las sesiones prácticas concretas, los puntos de interés sobre los que nos interesa reflexionar -el contacto corporal, la desinhibición, o el liderazgo, por ejemplo-, obligando de este modo al alumno a focalizar su atención en esos aspectos de la sesión, para desarrollar posteriormente su reflexión, en el diario, de forma más centrada y profunda. 
La observación del compañero es otra de las alternativas que proponemos para este tipo de trabajo: el alumno puede observar la actuación de un compañero, a nivel práctico y a nivel de destreza docente, buscando en el otro respuestas o actitudes que ellos consideren que no dominan, o de las que carecen.

\section{Bibliografía}

Camerino, O. y Buscá, F. (2001). La formación del docente en Educación Física: un modelo de formación reflexiva inicial y continuada. En B. Vázquez, (Coord.), Bases educativas de la actividad física y el deporte (pp. 227-240). Madrid: Síntesis.

Carreiro da Costa, F. (1996). Formaçao de Professores: Objectivos, Conteúdos e Estratégias. En F. Carreiro, M. Carvalho, M. Onofre, J. Diniz y C. Pestana, (Eds.), Formaçao de Professores em Educaçao Física. Concepçoes, investigaçao, prática (pp. 9-36). Lisboa: FMH-UTL.

Colás, M. P. (1992). La metodología cualitativa. En M. P. Colás y L. Buendía (Eds.), Investigación educativa (pp. 249-290). Sevilla: Ediciones Alfar. Piados.

Coll, C. (1987). Psicología y currículum. Barcelona:

Debesse, M. (1982). Un problema clave de la educación escolar contemporánea. En M. Debesse y G. Mialaret (Eds.), La formación de los enseñantes (pp. 13-34). Barcelona: Oikos-Tau.

Del Villar, F. (1993). El desarrollo del conocimiento práctico de los profesores de educación física, a través de un programa de análisis de la práctica docente. Un estudio de casos en formación inicial. Tesis Doctoral. Granada: Universidad de Granada.

Fraile, A. (1993). Un modelo de formación permanente para el profesorado de educación física. Tesis Doctoral. Valladolid: Universidad de Valladolid.

García Aretio, L. (1.989). La Educación. Teorías y Conceptos. Perspectiva Integradora. Madrid: Paraninfo.

Hopkins, D. (1989). Investigación en el aula. Barcelona: P.P.U.

Latorre, A., y González, R. (1987). El maestro investigador. Barcelona: Grao.
Marchesi, A. y Martín, E. (1999). Calidad de la enseñanza en tiempos de cambio. Madrid: Alianza Editorial.

Medina, J. (1995). Influencia de un entrenamiento docente basado en el trabajo en grupo durante la formación inicial del profesorado de educación física: un estudio preliminar. Tesis Doctoral. Granada: Universidad de Granada.

Moral, C. (1998). Formación para la profesión docente. Granada: Grupo FORCE y Grupo Editorial Universitario.

Moreno, P. y García, T. (1999). Investigación en la acción en el ámbito universitario: propuesta de utilización del diario personal en Expresión Corporal. Actas I Congreso Internacional de Educación Física: «La Educación Física en el siglo XXI» (pp. 731-738). Fondo Editorial de Enseñanza.

Pérez, A. (1992). Enseñanza para la comprensión. En J. Gimeno y A. Pérez (Eds.), Comprender la enseñanza (pp. 78-114). Madrid: Morata.

Pérez, A. (1995). Autonomía profesional del docente y control democrático de la práctica educativa. Actas del Congreso Internacional de Didáctica: "Volver a Pensar la Educación"(pp. 339-353). Madrid: Morata.

Pozo, I. (2001). Aprendices y maestros. La nueva cultura del aprendizaje. Madrid: Alianza Editorial.

Ramos, L. A. (1999). La evolución del pensamiento docente de los profesores de educación física a través de un programa de supervisión orientado a la reflexión en la acción y sobre la acción. Tesis Doctoral. Cáceres: Universidad de Extremadura.

Rodríguez, J. Ma . (1994). Las prácticas de enseñanza en la formación inicial de los profesores. Tesis Doctoral. Sevilla: Universidad de Sevilla.

Rodríguez, J. Ma. (1997). Bases y Estrategias de Formación Permanente del Profesorado. Huelva: Hergué.

Romero, C. (1995). Incidencias de un programa de formación inicial del maestro especialista en educación fisica en los niveles de reflexión y toma de decisiones sobre la práctica docente. Tesis Doctoral. Granada: Universidad de Granada.

Schön, D. A. (1983). The reflective practitioner. How proffesionals think in action. New York: Basic Books.

Sicilia, A. (1999). El diario personal del alumnado como técnica de investigación en Educación Física. Apunts, 58, 25-33. 